\title{
Subcutaneous Administration of Granulocyte- Colony Stimulating Factor Versus Local Infusion on IUI Outcomes in Women with Unexplained Infertility
}

\author{
Fatemeh Arab Baniasad ${ }^{1}$, Ensieh Salehi ${ }^{1}$, Nasibeh Roozbeh ${ }^{2}$, Leila Moalemzadegan ${ }^{1}$ and \\ Maryam Azizi Kutenaee ${ }^{1 *}$ \\ ${ }^{1}$ Fertility and Infertility Research Center, Hormozgan University of Medical Sciences, Iran \\ ${ }^{2}$ Mother and Child Welfare Research Center, Hormozgan University of Medical Science, Iran \\ *Corresponding author: Maryam Azizi Kutenaee, Fertility and Infertility Research Center, Hormozgan University of Medical \\ Sciences, Bandar Abbas, Iran
}

\section{ARTICLE INFO}

Received: May 01, 2021

Published: 㭡 May 07, 2021

Citation: Fatemeh Arab B, Ensieh Salehi, Nasibeh Roozbeh, Leila Moalemzadegan, Maryam Azizi Kutenaee. Subcutaneous Administration of Granulocyte- Colony Stimulating Factor Versus Local Infusion on IUI Outcomes in Women with Unexplained Infertility. Biomed J Sci \& Tech Res 35(4)2021. BJSTR. MS.ID.005733.

Keywords: G-CSF; Intrauterine Infusion; Subcutaneous; IUI; Unexplained Infertility

\section{ABSTRACT}

Purpose: To evaluate the effect of single-dose subcutaneous of G-CSF administration versus intrauterine infusion on IUI outcomes in patients with unexplained infertility.

Materials and Methods: This was a randomized clinical trial under tertiary setting at Infertility Clinic affiliated to Hormozgan University of Medical Sciences between Jun 2020 and Dec 2020. One hundred and thirty-nine eligible patients, on the day of human chorionic gonadotrophin (HCG) administration, were randomized into 3 groups :(A) patients received $300 \mu \mathrm{g}$ G-CSF subcutaneously, (B)in this group, 300 $\mu \mathrm{g}$ G-CSF was infused intrauterine, and $(\mathrm{C})$ patients received no G-CSF treatment. Biochemical pregnancy and clinical pregnancy were measured and compared between groups.

Result: The pregnancy outcomes in women who received G-CSF increased in comparison to the control $(\mathrm{p}<0.05)$. Moreover, the results of our study showed that the chemical pregnancy and clinical pregnancy were higher in women who received intrauterine G-CSF infusion versus subcutaneous administration (respectively, $24.4 \%$ vs $20 \%$ and $24.4 \%$ vs $15.55 \%$ ) but these differences were not statistically significant $(\mathrm{p}>0.05)$.

Conclusion: G-CSF can conceivably improve pregnancy outcomes. Intrauterine route appears to be more effective than subcutaneous. However, Further studies with higher sample size are needed to establish the best route of G- CSF administration in particular with regards to women with unexplained infertility undergoing IUI.

\section{Introduction}

Infertility is defined by the failure to achieve pregnancy after 12 months or more of regular intercourse without contraception methods [1,2]. Globally, it is estimated that $15 \%$ of couples of reproductive ages suffer from infertility and seek for medical help [3]. Among couples presenting for the standard infertility evaluation, approximately $30-50 \%$ of them have been diagnosed with unexplained infertility [4]. The exact etiology underlying unexplained infertility is still unclear and its treatment relies on empirical methods [5]. Although, overall pregnancy rate in couples with unexplained infertility is rather high, some couples fail to conceive after several attempts causing adverse effects on quality of life and a heavy financial burden [6].

Increasing the efficacy of treatments in infertile patients, the administration of G-CSF as an adjuvant has recently attracted more attention due to its key roles in implantation and pregnancy maintenance [7-9]. G-CSF is a member of CSF cytokine family produced by hematopoietic cells, macrophages, trophoblast, 
and NK cells [10]. G-CSF and its receptors are also expressed in reproductive tract, especially in follicular cells and at the maternalfetal interface during the time of implantation and early pregnancy [11]. This cytokine and other members of the CSF family are involved in preparing a local immune environment for a successful implantation. Further, G-CSF is associated with dendritic cell maturation, blastomere viability, and Th-2 cytokine secretion. Finally, G-CSF plays an important role in tolerance maintenance at the utero-placental interface through regulatory $\mathrm{T}$ cell activation $[11,10]$.

Based on the evidence that G-CSF contributed to implantation, several authors demonstrated that administration of G-CSF before implantation increased the IVF success, implantation, and pregnancy rate in women with unexplained recurrent miscarriage or implantation failure $[7,9,12]$. while, other studies did not find an improvement in implantation rate, or clinical pregnancy rate after ART with G-CSF administration [13,14]. However, large, randomized, controlled trials will help to overcome the discrepancies of G-CSF efficacy in infertile patients. The administration of G-CSF in patients with unexplained infertility undergoing IUI is less common and less extensively documented in literature. In addition, systemic administration of G-CSF is thought to be more effective than intrauterine infusion [13]. We conducted this investigation on patients with unexplained infertility to examine the efficacy of subcutaneous G-CSF administration versus local infusion on IUI success, biochemical, and clinical pregnancy rates.

\section{Materials and Methods}

This prospective randomized clinical trial study (IRCT20200303046688N1) was approved by the Ethics Committee of Hormozgan University of Medical Sciences (IR.HUMS REC.1398.443) and carried out in compliance with the Helsinki Declaration. In addition, signed informed consent was obtained from all patients prior to participation. The study was conducted on 200 patients aged $\leq 38$-year candidates for IUI due to unexplained infertility (consistent with the World Health Organization criteria) at Hormozgan university infertility center, between Jun 2020 and Dec 2020. All patients with hCG day endometrial thickness $<7 \mathrm{~mm}$, known causes of infertility, congenital or acquired uterine anomaly including polyp, myoma, intrauterine adhesions, presence of contraindications for G-CSF treatment (sickle cell disease, chronic neutropenia, renal insufficiency, present malignancy, pneumonia), and abnormal semen parameters were excluded from the study.

Approximately $12 \mathrm{~h}$ before hCG administration, all patients who met the inclusion criteria of the study were randomly divided into 3 groups: A ( $\mathrm{n}=47)$; $300 \mu \mathrm{g}$ G-CSF $(0.5 \mathrm{ml})$ (Neupogen, Roche) subcutaneously administrated, B $(n=47) ; 300 \mu \mathrm{g}$ G-CSF was administered into the fundus of the uterus using an IUI catheter, and $\mathrm{C}(\mathrm{n}=45)$; the cycle were continued without G-CSF infusion
Randomization was done by a computer-generated randomization block table. Controlled ovarian stimulation was performed with combination of letrozole and rFSH (Gonal-f; Merck Serono). Briefly, all recruited patients received letrozole (Iran hormone, Tehran, Iran) at a dose of $2.5 \mathrm{mg}$ twice a day for 5 days from day 3 of menses. Intramuscular injection of Gonal-f 150 IU was done every day starting from day 8.

When there was at least one follicle $\geq 18 \mathrm{~mm}$ (average of diameter measured in three dimensions), 5000 IU hCG (Ovitrelle, Merck Serono) was injected subcutaneously to trigger ovulation. $36 \mathrm{~h}$ later, IUI was performed, and all patients received luteal phase support as $90 \mathrm{mg} /$ day of Crinone gel (Merck Serono, UK) starting on the day of IUI. Biochemical pregnancy was evaluated two weeks later, and clinical pregnancy was defined as the presence of a gestational sac on ultrasound performed 5 weeks after hCG injection. Statistical analysis was performed using the SPSS, V.16 (SPSS Inc., Chicago, IL, US). The distribution of data was analyzed by Kolmogorov-Smirnov test. Data were expressed as mean \pm standard deviation (SD) and percentage. Continuous and categorical variables were compared between groups by one-way analysis of variance (ANOVA) and the chi-square test. $\mathrm{p}$ values $<0.05$ were considered to be statistically significant.

\section{Result}

The Consolidated Standards of Reporting Trials (CONSORT) diagram of the participants was shown in Figure 1. In total, 200 women with unexplained infertility were evaluated for participation in this study. Of these, 61 patients were excluded because of non-eligibility for entering the study. The eligible women (139 patients) were randomly assigned to three groups including: subcutaneously G-CSF administration group (A, $n=47)$, intrauterine G-CSF infusion ( $B, n=47$ ), and control group (C, n=45). Two women from $B$ group refused to receive allocated intervention due to personal reasons and two women in A group did not inform the pregnancy tests. Finally, the cycle outcomes of 135 patients in three groups were compared. The demographic characteristics are presented in Table 1. There were no significant differences among study groups regarding age, BMI, FSH, TSH, PRL, and number of follicles $(p>0.05)$. The biochemical pregnancy and clinical pregnancy were significantly different among group $(\mathrm{p}=0.048, \mathrm{p}=$ 0.044 , respectively) (Table 2 ).

In addition, Table 3 compares the pregnancy outcome of the two groups to whom G-CSF was administered as local infusion or subcutaneous injection. The chemical pregnancy rate was apparently higher in group with local infusion than subcutaneous group $(24.4 \%$ vs $20 \%, \mathrm{p}=0.460)$. The clinical pregnancy rate was also higher in the group with local infusion than subcutaneous group (24.4\% vs $15.55 \%$, $\mathrm{p}=0.322$ ) but these differences were not statistically significant $(\mathrm{p}>0.05)$. 


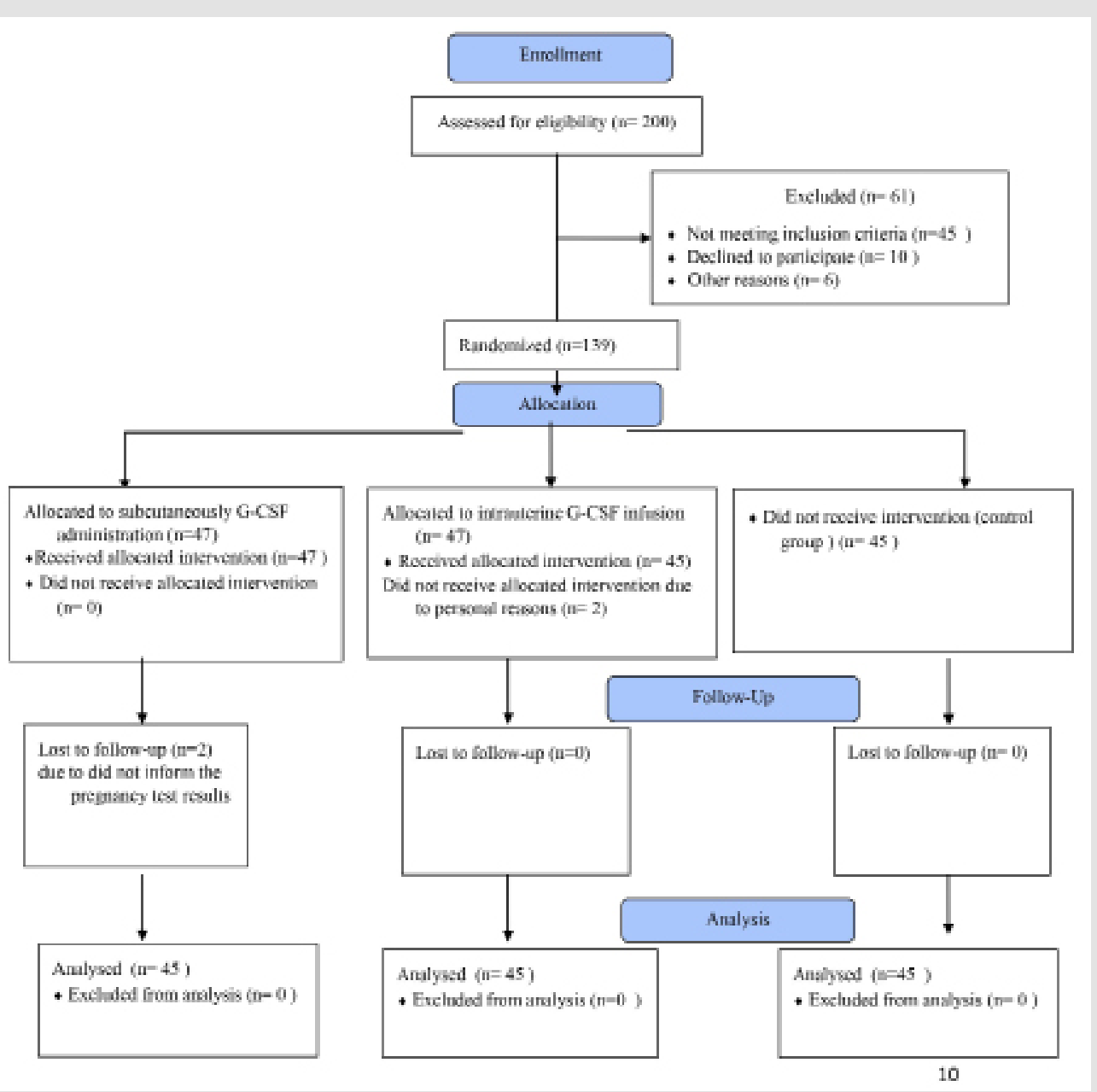

Figure 1: The study flowchart.

Table 1: Comparison of demographic data between groups.

\begin{tabular}{|c|c|c|c|c|}
\hline Variables & $\begin{array}{c}\text { Group A (SC } \\
\text { Administration) }\end{array}$ & $\begin{array}{c}\text { Group B (IU } \\
\text { Administration) }\end{array}$ & Group C (Without G-CSF) & p Value \\
\hline Age(years) & $27.96 \pm 4.46$ & $28.42 \pm 5.63$ & $26.96 \pm 4.33$ & 0.505 \\
\hline BMI (kg/m2) & $23.6 \pm 1.66$ & $22.68 \pm 2.69$ & $22.87 \pm 1.52$ & 0.433 \\
\hline FSH (mIU/ml) & $5.34 \pm 2.18$ & $4.91 \pm 2.99$ & $4.81 \pm 1.95$ & 0.692 \\
\hline PRL (ng/ml) & $133.53 \pm 179.57$ & $82.61 \pm 111.50$ & $126.12 \pm 128.75$ & 0.429 \\
\hline TSH (mIU/ml) & $2.28 \pm 1.15$ & $2.51 \pm 0.84$ & $2.69 \pm 1.18$ & 0.383 \\
\hline Number of follicles & $2.12 \pm 0.93$ & $1.82 \pm 0.98$ & $1.83 \pm 0.83$ & 0.413 \\
\hline
\end{tabular}

Table 2: Comparison of reproductive outcomes between groups after intervention.

\begin{tabular}{|c|c|c|c|c|}
\hline Variables & $\begin{array}{c}\text { Group A (SC } \\
\text { Administration) }\end{array}$ & $\begin{array}{c}\text { Group B (IU } \\
\text { Administration) }\end{array}$ & Group C (Without G-CSF) & P Value \\
\hline Biochemical pregnancy & $9(20 \%)$ & $11(24.4 \%)$ & $6(13.3 \%)$ & $0.048^{*}$ \\
\hline Clinical pregnancy & $7(15.55 \%)$ & $11(24.4 \%)$ & $3(6.66 \%)$ & $0.044^{*}$ \\
\hline
\end{tabular}

Note: Data are presented as n (\%). IU: Intrauterine, SC: Subcutaneous; *Significance values 
Table 3: Comparison of reproductive outcomes between two intervention routes.

\begin{tabular}{|c|c|c|c|}
\hline Variable & Group 1 (SC Administration) (n=45) & Group2 (IU Administration) (n=45) & P Value \\
\hline Biochemical pregnancy & $9(20 \%)$ & $11(24.4 \%)$ & 0.46 \\
\hline Clinical pregnancy & $7(15.55 \%)$ & $11(24.4 \%)$ & 0.322 \\
\hline
\end{tabular}

Note: Data are presented as n (\%). IU: Intrauterine, SC: Subcutaneous

\section{Discussion}

Unexplained infertility refers to failure to achieve a pregnancy without an obvious cause [4]. Although the exact etiology underlying unexplained infertility is still unclear but inadequate endometrial receptivity is thought to be related to the etiology of unexplained infertility [15]. Therefore, identifying key factors that are involved in endometrial remodeling and implantation process could provide a promising tool in treatment of women with unexplained infertility or implantation failure. These observations that serum level of G-CSF continuously increases from the day of embryo transfer to the day of implantation and from the day of confirmation of pregnancy to gestation [16], and infertile women with implantation failure express the lower level of G-CSF receptors at the maternal-fetal interface [17] attracted the attentions toward this glycoprotein with growth factor and cytokine functions. Evidence have illustrated that G-CSF is involved in the following events during implantation and pregnancy which include.

a) Trophoblast proliferation and invasion.

b) Blastocyst growth and development.

c) Embryo-endometrial interaction, and

d) Modulation of essential genes involved in embryo attachment, apoptotic activity, cell migration, tissue and vascular remodeling [17].

G-CSF has also been found to promote maternal tolerance to the semi-allogenic embryo through shifting the inflammatory balance towards an anti-inflammatory Th2 response, T regulatory cells enhancement, and tolerogenic dendritic cell differentiation [18]. Due to the safety of G-CSF application in pregnancy [19] and no reported major side effects, Gleicher used the G-CSF for the first time in treatment of four unresponsive women with thin endometrium, and a pregnancy was achieved in all four patients [20]. since that, several clinical trials have conducted to evaluate the effect of G-CSF on IVF outcomes [9,12,7]. Some investigators have demonstrated the effectiveness of G-CSF administration in improving the reproductive outcomes in IVF / ICSI / FET cycles [9, 21,22], even though, others found no treatment benefit of G-CSF in assisted reproductive technology (ART) $[13,14,23]$. To give a better answer to this topic under debate, a recent meta-analysis study in women with unexplained, repeated implantation failure (RIF) revealed the favorable effect of G-CSF on the implantation rate and clinical pregnancy rate, especially when administrated by subcutaneous injection [24]. However, other published systematic review that included 15 trials containing 1253 sub fertile women undergoing assisted reproduction declared the administration of G-CSF is effective only in women with two or more previous IVF failure history [25], and its effectiveness in other sub fertile women is unclear.

At present, scientific data regarding the application of G-CSF and the best route of its administration for infertile women trying to get pregnant through IUI is very limited. In present study, we found that G-CSF improves the biochemical and clinically pregnancy rates in patients with unexplained infertility after IUI compared to control. Our findings are in consistence with those from the only previous RCT in IUI cycle [26] which reported that the subcutaneous G-CSF injection is associated with the improvement in implantation and pregnancy rate. However, their results were not statistically significant. Since two studies are very similar in many characteristics such as selected patient population, dose of G-CSF administration, type of infertility, having normal endometrial thickness, and stimulated protocol, it seems that the difference in time of administration (on trigger day in current study versus two days after IUI) has affected the results.

In a follow up study carried out by Gleicher et al., 21patients with unresponsive thin endometrium resistant to standard therapies were treated with intrauterine G-CSF instillation, resulting in significant endometrial expansion and a 19.1\% ongoing pregnancy rate [22]. Another study by Kalem et al. intrauterine G-CSF administration in RIF patients with normal endometrial thickness were investigated, and it was reported that the intervention of G-CSF administration did not change the endometrial thickness, clinical pregnancy rates, or live birth rates. However, this study is not comparable to our study because of difference in selected population, dose of G-CSF, and stimulation protocol. The duration and time of drug administration, G-CSF dosage, the route of administration, the patient age, and the cause of infertility are thought to be the main factors which can impact the results. Additionally, we observed the local infusion of G-CSF seems to be more effective than subcutaneous administration, however our data was not statistically significant. In contrast, a most recently meta- analysis by Jiang et al. has concluded that both intrauterine and subcutaneous injection of G-CSF can improve implantation and clinical pregnancy rate, but the subcutaneous injection appears to be superior to intrauterine instillation for unexplained RIF patients. We cannot accurately estimate the reasons behind our observation regarding the route of administration, but at least, we speculate intrauterine route can provide a desire environment around 
the implantation site by creating a higher local concentration. However, it is important to notice that there is a lack of data on the straight comparison between different routes of administration in patients with normal endometrium undergoing IUI and this issue can be considered as a strength of current study. Further, the main limitations of our study were absence of blinding due to the nature of the study. Moreover, no placebo was involved in the research, therefore, we cannot omit the endometrial injury effect that could potentially influence the results of the study. In addition, we did not evaluate the live birth rate and miscarriage in all groups that could help to interpret the results with more validity and establishing the safety of G-CSF administration.

\section{Conclusion}

Our results provided further support for the beneficial effect of G-CSF on pregnancy outcomes in women with unexplained infertility undergoing IUI, especially when administrated intrauterine. However, no evidence favoring a specific route of G-CSF administration in women with unexplained fertility and normal endometrial thickness was found, and further trial studies with larger sample size are needed to elucidate this issue.

\section{Acknowledgement}

The authors wish to thank all the patients who participated in the study and are grateful to Hormozgan University of Medical Sciences for financial support.

\section{Declarations}

\section{Ethics approval and consent to participate}

This study was approved by the Ethics Committee of Hormozgan University of Medical Sciences (IR.HUMS.REC.1398.443) and signed informed consent was obtained from all patients prior to participation.

\section{Competing Interests}

The authors declare that they have no competing interests.

\section{Funding}

This study was supported by the research fund of Hormozgan University of Medical Sciences (grant number 443).

\section{Authors' Contributions}

MAK: conceived, designed and monitored the study. FAB: conducted the study and performed the analysis. ES: Manuscript writing/editing, data analysis. NR: project development. LM: data collection. All authors read and approved the final manuscript.

\section{References}

1. Zegers-Hochschild F, Adamson GD, Dyer S, Racowsky C, De Mouzon J, Sokol R, et al. (2017) The international glossary on infertility and fertility care, 2017. Human reproduction 32 (9): 1786-1801.
2. Rahbar A, Abbasi M (2020) A Brief Clinical Overview of Etiological Factors in Infertility. Journal of Infertility and Reproductive Biology 8: 6-8.

3. Agarwal A, Mulgund A, Hamada A, Chyatte MR (2015) A unique view on male infertility around the globe. Reproductive biology and endocrinology 13 (1): 37.

4. Buckett W, Sierra S (2019) The management of unexplained infertility: an evidence-based guideline from the Canadian Fertility and Andrology Society. Reproductive biomedicine online 39 (4): 633-640.

5. Ray A, Shah A, Gudi A, Homburg R (2012) Unexplained infertility: an update and review of practice. Reproductive biomedicine online 24 (6): 591-602.

6. Brandes M, Hamilton C, Van der Steen J, De Bruin J, Bots R, et al. (2011) Unexplained infertility: overall ongoing pregnancy rate and mode of conception. Human Reproduction 26 (2): 360-368.

7. Eftekhar M, Miraj S, Mojtahedi MF, Neghab N (2016) Efficacy of intrauterine infusion of granulocyte colony stimulating factor on patients with history of implantation failure: A randomized control trial. International Journal of Reproductive Biomedicine 14 (11): 687.

8. Scarpellini F, Sbracia M (2009) Use of granulocyte colony-stimulating factor for the treatment of unexplained recurrent miscarriage: a randomised controlled trial. Human Reproduction 24 (11): 2703- 2708.

9. Scarpellini F, Sbracia M (2012) G-CSF treatment improves IVF outcome in women with recurrent implantation failure in IVF. Journal of Reproductive Immunology 1 (94): 103.

10. Alijotas-Reig J, Llurba E, Gris JM (2014) Potentiating maternal immune tolerance in pregnancy: a new challenging role for regulatory $\mathrm{T}$ cells. Placenta 35 (4): 241-248.

11. Rahmati M, Petitbarat M, Dubanchet S, Bensussan A, Chaouat G, et al. (2015) Colony stimulating factors $1,2,3$ and early pregnancy steps: from bench to bedside. Journal of reproductive immunology 109: 1-6.

12. Zafardoust S, Akhondi MM, Sadeghi MR, Mohammadzadeh A, Karimi A, et al. (2017) Efficacy of intrauterine injection of granulocyte colony stimulating factor (G-CSF) on treatment of unexplained recurrent miscarriage: a Pilot RCT study. Journal of Reproduction \& Infertility 18 (4): 379.

13. Barad DH, Yu Y, Kushnir VA, Shohat-Tal A, Lazzaroni E, et al. (2014) A randomized clinical trial of endometrial perfusion with granulocyte colony-stimulating factor in in vitro fertilization cycles: impact on endometrial thickness and clinical pregnancy rates. Fertility and sterility 101 (3): 710-715.

14. Li Y, Pan P, Chen X, Li L, Li Y, et al. (2014) Granulocyte colony-stimulating factor administration for infertile women with thin endometrium in frozen embryo transfer program. Reproductive Sciences 21 (3): 381385.

15. Altmäe S, Martinez-Conejero J, Salumets A, Simon C, Horcajadas J, et al. (2009) Endometrial gene expression analysis at the time of embryo implantation in women with unexplained infertility. MHR: Basic science of reproductive medicine 16 (3): 178-187.

16. Salmassi A, Schmutzler A, Schaefer S, Koch K, Hedderich J, et al. (2005) Is granulocyte colony-stimulating factor level predictive for human IVF outcome? Human Reproduction 20 (9): 2434-2440.

17. Rahmati M, Petitbarat M, Dubanchet S, Bensussan A, Chaouat G, et al (2014) Granulocyte-colony stimulating factor related pathways tested on an endometrial ex-vivo model. PloS one 9 (10): e102286.

18. Leone G, Rutella S, Zavala F, Danese S, Kared H (2005) Granulocyte Colony-Stimulating Factor: A J Immunol 175: 7085-7091.

19. Dale DC, Cottle TE, Fier CJ, Bolyard AA, Bonilla MA, et al. (2003) Severe chronic neutropenia: treatment and follow-up of patients in the Severe 
Chronic Neutropenia International Registry. American journal of hematology 72 (2): 82-93.

20. Gleicher N, Vidali A, Barad DH (2011) Successful treatment of unresponsive thin endometrium. Fertility and sterility 95 (6): 2123. e2113-2123. e2117.

21. Aleyasin A, Abediasl Z, Nazari A, Sheikh M (2016) Granulocyte colony-stimulating factor in repeated IVF failure, a randomized trial. Reproduction (Cambridge, England) 151 (6): 637.

22. Gleicher N, Kim A, Michaeli T, Lee H, Shohat-Tal A, et al. (2013) A pilot cohort study of granulocyte colony-stimulating factor in the treatment of unresponsive thin endometrium resistant to standard therapies. Human Reproduction 28 (1): 172-177.

\section{ISSN: 2574-1241}

DOI: $10.26717 /$ BJSTR.2021.35.005733

Maryam Azizi Kutenaee. Biomed J Sci \& Tech Res

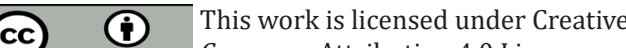

Submission Link: https://biomedres.us/submit-manuscript.php
23. Kalem Z, Kalem MN, Bakirarar B, Kent E, Makrigiannakis A, et al. (2020) Intrauterine G-CSF Administration in Recurrent Implantation Failure (RIF): An Rct. Scientific Reports 10 (1): 1-7.

24. Jiang Y, Zhao Q, Zhang Y, Zhou L, Lin J, et al. (2020) Treatment of G-CSF in Unexplained, Repeated Implantation Failure: A Systematic Review and Meta-analysis. Journal of Gynecology Obstetrics and Human Reproduction: 101866.

25. Kamath MS, Kirubakaran R, Sunkara SK (2020) Granulocyte-colony stimulating factor administration for subfertile women undergoing assisted reproduction. Cochrane Database of Systematic Reviews (1).

26. Aramesh S, Kutenaee MA, Najafi F, Ghafari P (2020) Effect of granulocyte colony stimulating factor on pregnancy rate in women with unexplained infertility after intrauterine insemination: a randomized clinical trial.

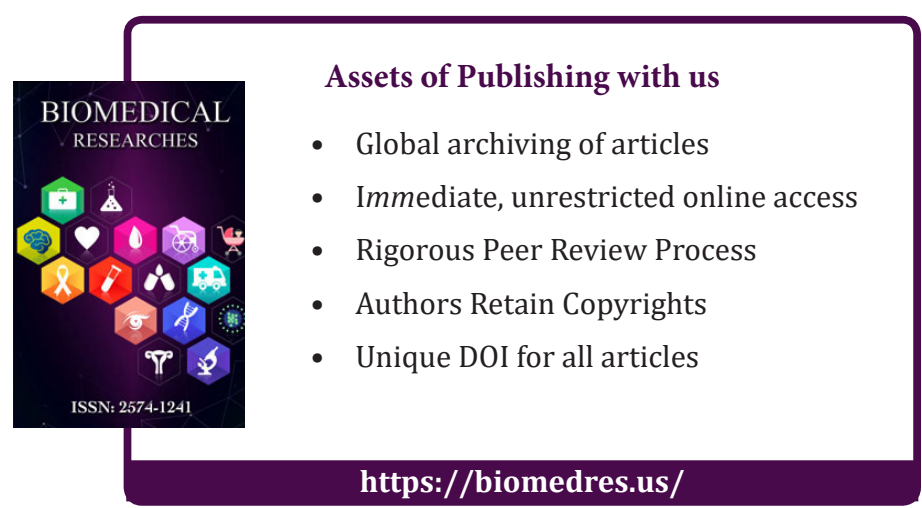

